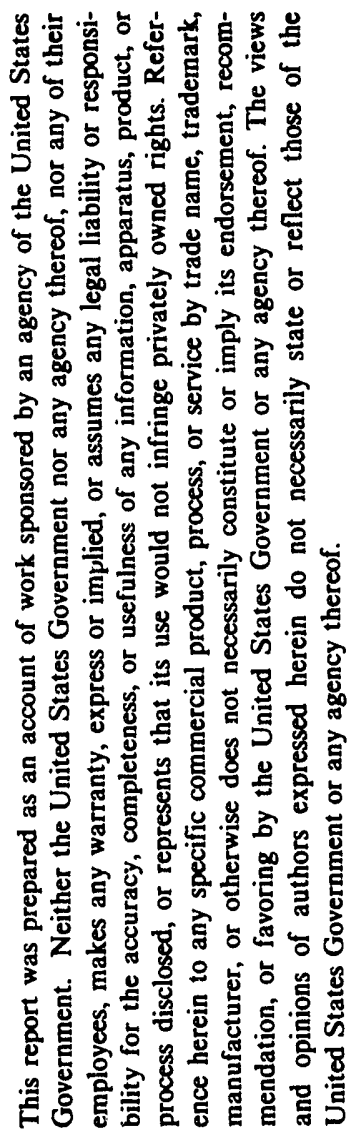

\author{
FINAL PROGRESS REPORT \\ BUDGET PERIOD \\ MAY 1, 1990 - DECEMBER 15, 1992 \\ John M. Davis and Stephen K. Cox \\ Department of Atmospheric Science \\ Colorado State University \\ Fort Collins, CO 80523
}

December 15, 1992

Prepared for

THE US DEPARTMENT OF ENERGY AGREEMENT NO. DE-FG02-90ER60970
RECEIVED JUN 071993 OSTI 


\title{
Monitoring the Response of the Upper Troposphere/Lower Stratosphere to a Greenhouse Gas Scenario
}

\begin{abstract}
During the past two years a system has been under development to contribute to this monitoring process by making measurement of the downwelling infrared radiance field at moderate spectral resolution. Sensitivity of the measurements to the presence of cirrus cloudiness, and effective particle size in cirrus clouds has been demonstrated. Sensitivity of the measurements to concentration of $\mathrm{CO}_{2}$ and to the gross temperature structure of a $2 \mathrm{CO}_{2}$ atmosphere have been modeled, and measurements have been made at various locations to provide a data base for further system evaluation. A temperature retrieval algorithm has been acquired and examined concerning its potential for monitoring temperature and gaseous concentration profiles. A continued research program is described herein that may be divided into three parts. First, continued system development must be pursued. This includes continued attention to the absolute accuracy of the measurements, and implementation of the Path Characterization Algorithm (retrieval code). Second, the value of long term deployment of the system at a site of considerable elevation is necessary. Third, inclusion of a solar transmission capability at moderate resolution is proposed.
\end{abstract}

\section{NOTICE}

This report was prepared as an account of work sponsored by the United States Government. Neither the United States nor the Department of Energy, nor any of their employees, nor any of their contractors, subcontractors, or their employees, makes any warranty, express or implied, or assumes any legal liability or responsibility for the accuracy, completeness, or usefulness of any information, apparatus, product or process disclosed or represents that its use would not infringe privately-owned rights.

Copyright Notice. By acceptance of this article, the publisher and/or recipient acknowledges the U. S. Government's right to retain a nonexclusive, royalty-free license in and to any copyright covering this paper. 
Table of Contents

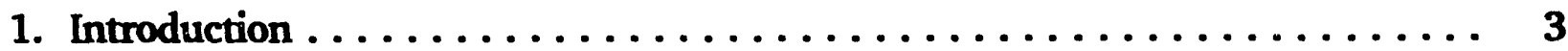

2. Summary of Research Objectives $\ldots \ldots \ldots \ldots \ldots \ldots \ldots \ldots \ldots \ldots, 3$

3.0 Progress Toward Research Objectives .................... 4

3.1 Interferometer System and Calibration $\ldots \ldots \ldots \ldots \ldots \ldots \ldots \ldots \ldots$

3.2 Instrument Deployments $\ldots \ldots \ldots \ldots \ldots \ldots \ldots \ldots \ldots \ldots \ldots, 9$

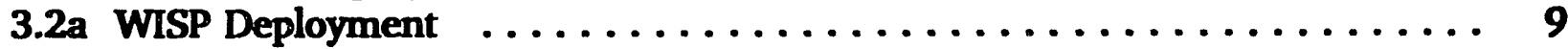

3.2b Pingree Park Deployment $\ldots \ldots \ldots \ldots \ldots \ldots \ldots \ldots \ldots \ldots \ldots, 10$

Zenith Angle Dependence $\ldots \ldots \ldots \ldots \ldots \ldots \ldots \ldots \ldots \ldots \ldots \ldots, 10$

Cirrus Cloud Detection $\ldots \ldots \ldots \ldots \ldots \ldots \ldots \ldots \ldots \ldots \ldots \ldots 12$

3.2c Fire IFO Deployment $\ldots \ldots \ldots \ldots \ldots \ldots \ldots \ldots \ldots \ldots \ldots \ldots \ldots$

Estimation of Effective Particle Size $\ldots \ldots \ldots \ldots \ldots \ldots \ldots \ldots \ldots 15$

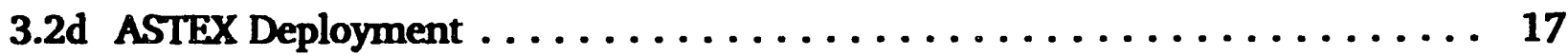

3.3 Temperature Retrieval Algorithm $\ldots \ldots \ldots \ldots \ldots \ldots \ldots \ldots \ldots \ldots \ldots$ 18

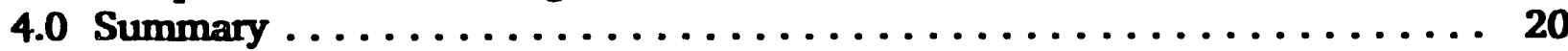

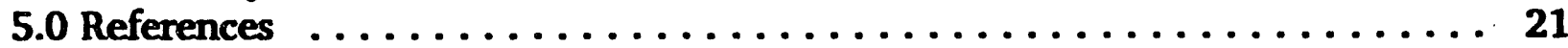

Rrepint temosed 


\section{Significant Research Accomplishments}

A system capable of monitoring the signals of a climate change scenario by remotely sensing the upper troposphere/lower stratosphere from a high altitude deployment has been developed. The system consist of a dual port emission interferometer, a calibration table with pointing mirror, and a high speed work station with a path characterization algorithm and line by line transmission code. Two blackbody systems were designed and tested to provide necessary calibration targets for the interferometer. The interferometer was deployed during four different periods at climatologically distinct locations to test the capabilities of the system. Over two hundred spectra have been gathered of in a variety of conditions ranging from a clear middle latitude atmosphere viewed from mountain site at $3.0 \mathrm{~km}$ ASL to sub-tropical marine stratocumulus cloudiness measured at Porto Santo Island near Madeira. The line by line radiance and transmission codes FASCOD2 and FASCOD3 were acquired to provide theoretical comparisons with the measured data. A state of the art computer work station was acquired in order facilitate the line by line calculations and to perform temperature retrievals. A retrieval algorithm formally dubbed "The Path Characterization Algorithm" was obtained from the Phillips Laboratory and was adapted to a pre-beta version of FASCOD3. The algorithm has been used successfully to retrieve temperature profiles using simulated measurements. Routines have been developed to introduce instrument effects to modeled spectra as a step toward implementation with real data. The interferometric data have been shown to be quite sensitive to the presence of cirrus clouds and have been used to estimate the size of "equivalent spherical" ice particles in cirrus clouds.

\section{Introduction}

This report summarizes the original research goals and the progress made toward those goals over the period from October, 1990 to the present. During this period significant resources have been expended toward developing an instrument system capable of monitoring the atmosphere to detect signals of climate change that may result from the increase in concentration of GGs (Greenhouse Gases). Results to date indicate that the performance of the hardware is near to original expectations; some questions on the degree of the absolute accuracy of the system remain. Software development is nearing completion with current activity focused on the use of the Path Characterization Algorithm for temperature and constituent concentration retrieval. Yet, it is not possible to determine if the monitoring system will provide the needed data without further long term deployment at a high altitude site.

\section{Summary of Research Objectives}

The complete statement of the research objectives of this project may be found in Appendix A, which is a copy of the original proposal. Basically, the objective is to develop a hardware/software system capable of monitoring the signals of a climate 
change scenario that might be caused primarily by an increase in $\mathrm{CO}_{2}$. Several modeling efforts in the literature qualitatively agree on the atmosphere's adjustment to a marked increase in the concentration of the gas. The original proposal utilized in particular the results (temperature and moisture adjustments to a $2 \mathrm{CO}_{2}$ atmosphere) in Rind (1987), to model atmospheric spectra in a clear atmosphere as measured from a high altitude tropical site. It was determined that the ratios of downwelling radiances measured at $60^{\circ}$ zenith to those at $0^{\circ}$ zenith were sensitive especially to the concentration of $\mathrm{CO}_{2}$ and to a lesser extent to the modeled temperature changes in the $2 \mathrm{CO}_{2}$ atmosphere, and that the ratio of radiances observed at zenith in a $2 \mathrm{CO}_{2}$ atmosphere to those in a standard atmosphere are sensitive anticipated temperature changes, although to a limited extent.

In addition to the ratio methods, it was proposed that more traditional temperature retrieval algorithms be examined for their applicability to the monitoring problem. The ability to retrieve profiles of water vapor and other Ggs concentrations would of course enhance our ability to detect signals of climate change since the concentration of $\mathrm{H}_{2} \mathrm{O}$ is subject to modification in a $2 \mathrm{CO}_{2}$ atmosphere according to climate models.

Finally, the interferometric system was proposed as a very sensitive detector of cloudiness and thus of changes over time of cloud behavior. For example, it is possible to estimate under various simplifying assumptions, the equivalent spherical radius of cloud particles for cirrus cloudiness. The interferometric signal is also an excellent signal of cloud base height when compared to clear sky spectra at the same site. Thus, a third primary signal of climate change, i.e. changes in cloud coverage, and on a first order basis, changing cloud type would be available.

\subsection{Progress Toward Research Objectives}

The preceding section outlined briefly the major research goals for the project. The following is a rather detailed account of the activity toward accomplishing these goals.

\subsection{Interferometer System and Calibration}

A Bomem MB100 Michelson interferometer was obtained in October, 1990. The instrument was configured with the dual port emission option which is schematically represented in Fig. 1.

This configuration allows the instrument to measure difference interferograms which result from the subtraction of a black body interferogram at near ambient temperature from that of the atmosphere, thus subtracting much of the contribution from the instrument itself. The optics allow a spectral response in the range from 350 to $6000 \mathrm{~cm}$. ${ }^{1}$ range but the measurements are limited by the spectral response of the middle infrared MCT detector to from 500 to $2000 \mathrm{~cm}^{-1}$. The detector is cooled cryogenicaily by 


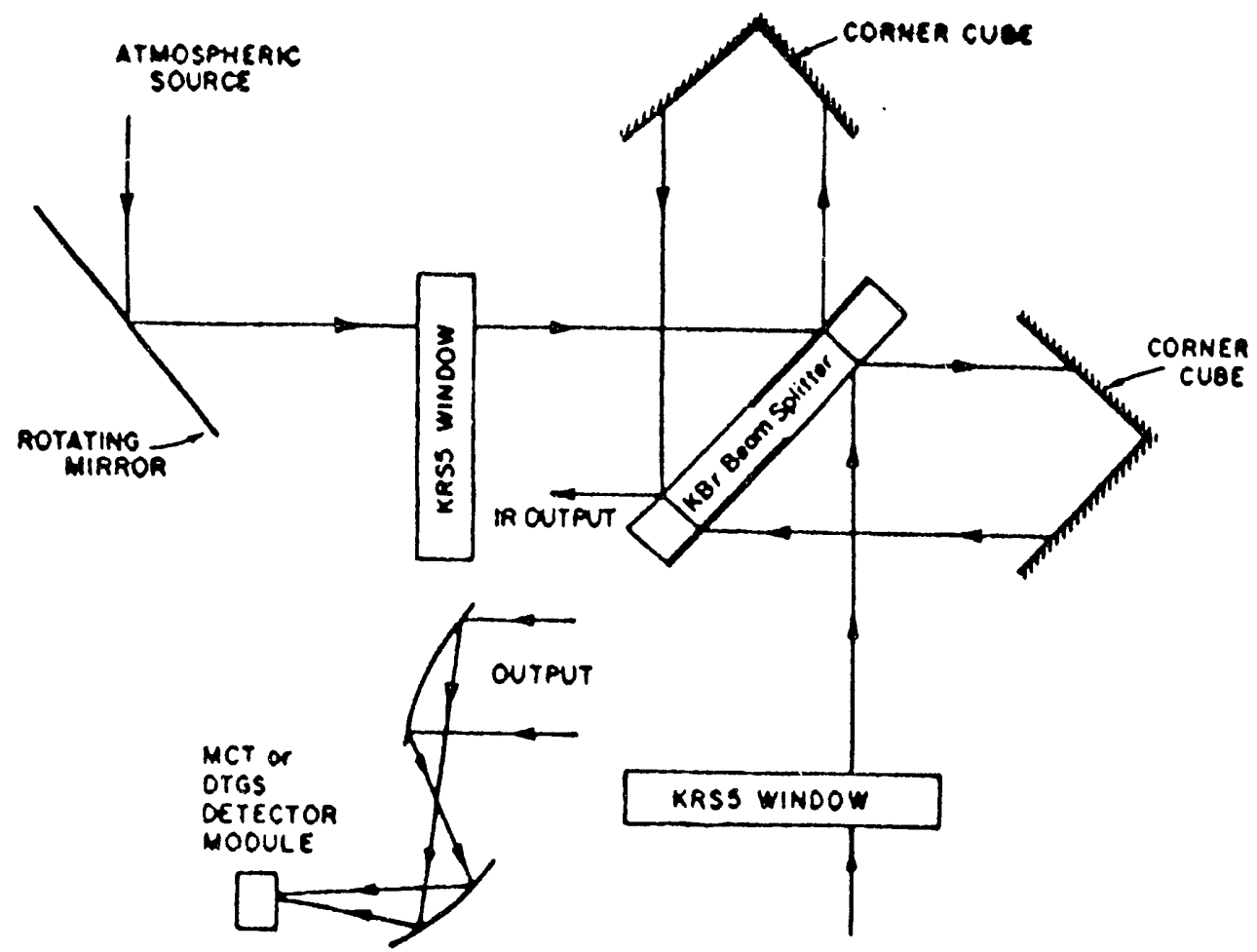

REFEACNCE SOURCE

Figure 1. Schematic diagram of the optical path of the somem
MB 100 dual port emission interferometer. expanding nitrogen gas into a small dewar behind the detector.

Originally, it was decided that the system should require as little operator interaction as possible since it was to be deployed at a site where operators were not necessarily intimately familiar with the system. With this in mind, the first calibration blackbodies utilized thermoelectric devices to cool copper disks to as low a temperature as possible. Fig. 2 shows one of the blackbodies under construction. Temperatures as low as $-50 \mathrm{C}$ were obtained by this method. The cold and warm blackbody sources were mounted on small table along with a gold mirror mounted on a DC servo motor whose shaft rotation could be directed with computer software to an angular resolution of 0.18 degrees. 


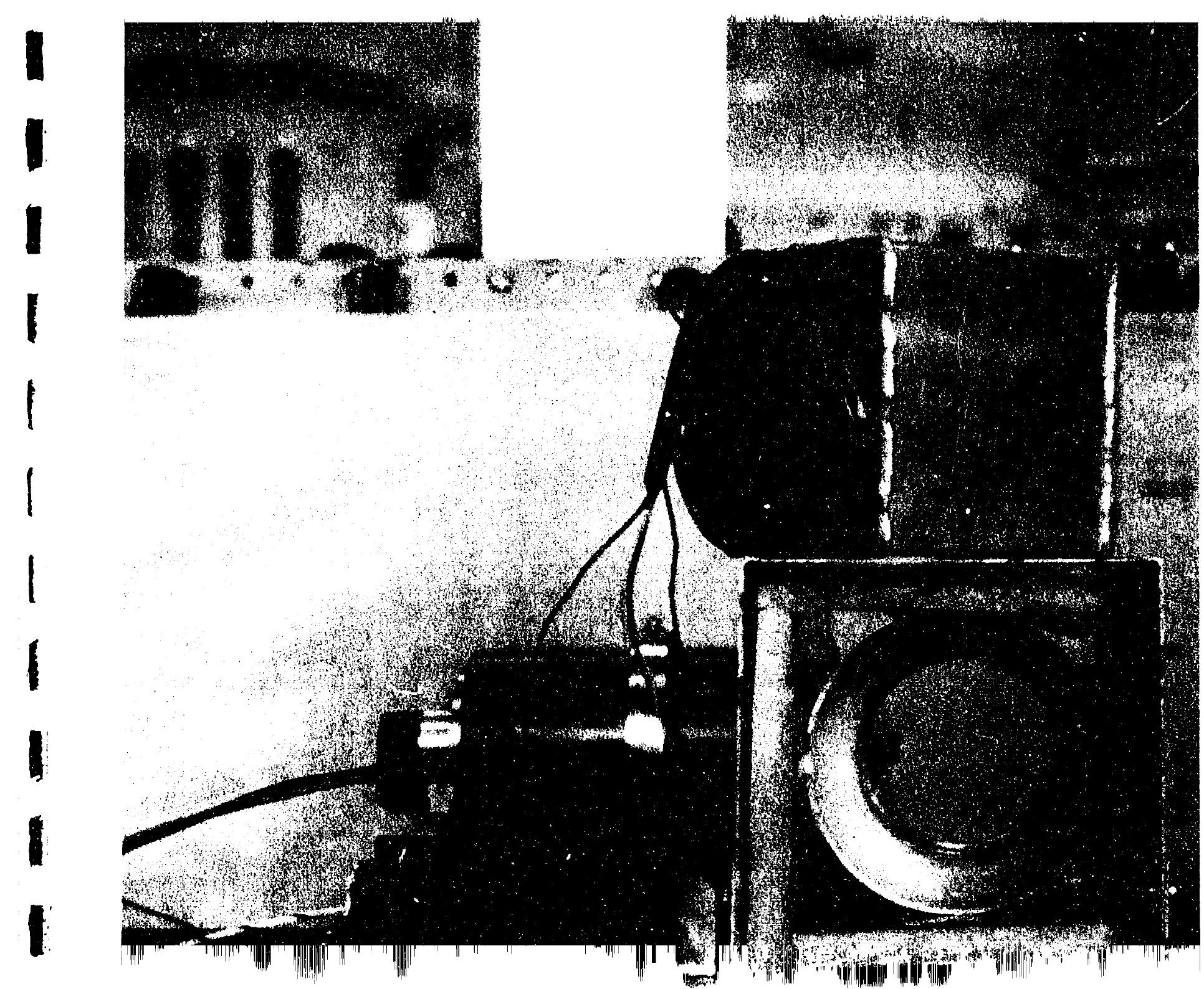


The calibration of the spectra followed the methodology of Revercomb et al (1988), without phase correction. Actually, phase correction of individual spectra is accomplished by the commercial software used to process the spectra according to the Mertz method; see Griffiths and de Haseth, (1986). In this method individual spectra are converted from interferograms by first applying a phase correction, triangularly apodizing the phase corrected interferogram and finally smoothing the data by zero filling before applying the Fast Fourier Transform converting the interferogram into a spectrum. An example of a test of the calibration procedure in which the measured spectrum of a laboratory black body is compared to the Planck function may be found in Fig. 5 of Appendix B, which also details the calibration procedure. Eventually it was found that the problem of frost deposition on the cooled disk outweighed the convenience of the thermoelectrically cooled system and a liquid nitrogen bath was adopted for use as the cold blackbody target. When the liquid nitrogen bath replaced the thermoelectrically cooled disk a conical copper black body was installed as the warm black body target. Fig. 3 shows the current configuration of the system. The copper black body is seen toward the rear, the LN2 dewar is positioned below the table. The interferometer views the LN2 source, the copper black body and the atmosphere by looking at their reflection from a gold coated mirror. The path to the LN2 dewar is protected as much as possible by a pipe above the lid of the dewar to lessen the effects of "cloud" formation in the cold air above the top of the dewar. 


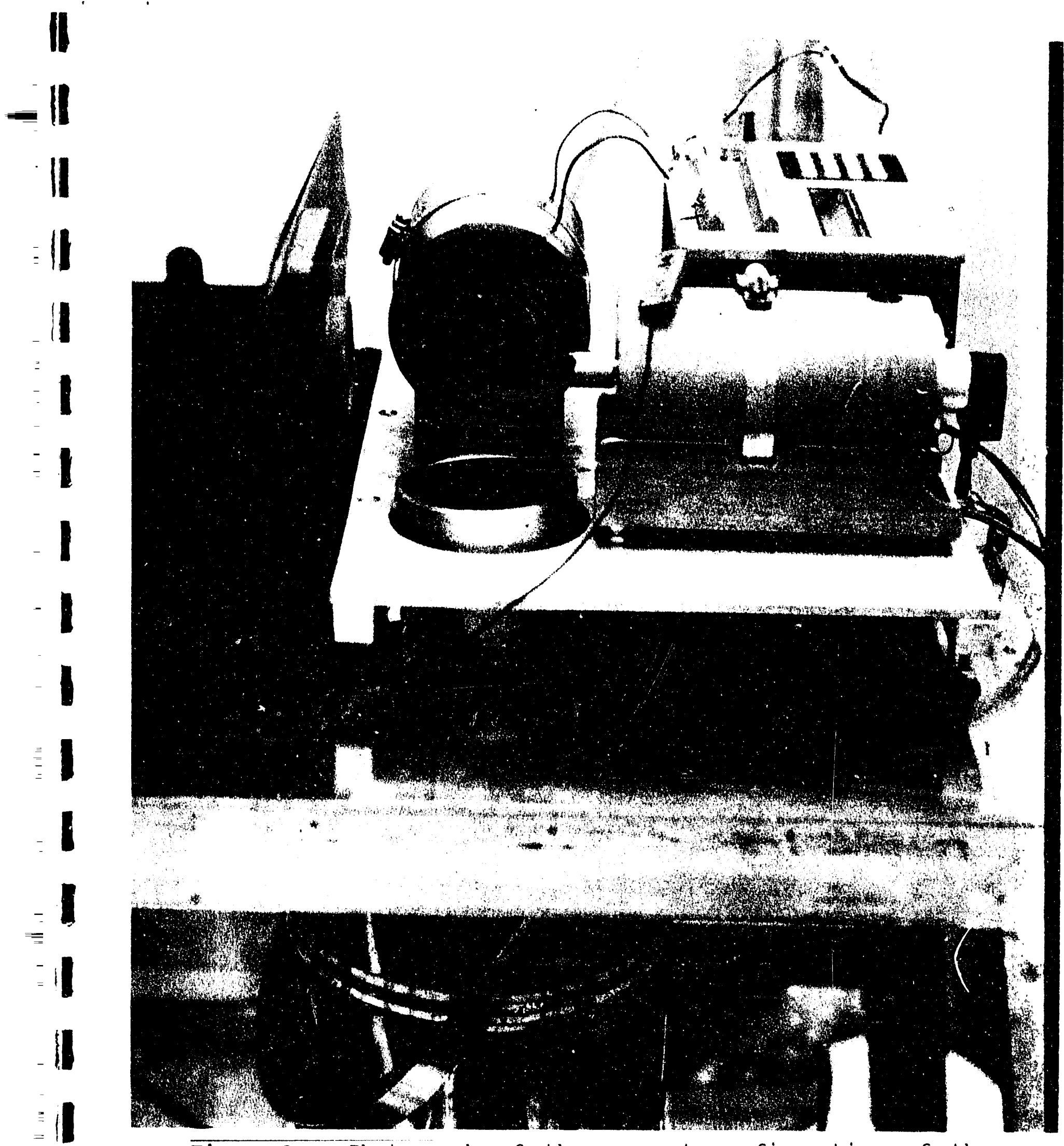

Figure 3. Photograph of the current configuration of the interferometer system.

trace gas monitoring system. Proceedings of conference entitled, "Remote Sensing of 


\subsection{Instrument Deployments}

One of the primary goals of this research was to demonstrate the ability to collect data at a high elevation site preferably in the tropics. At the time of the writing of the original proposal the Mauna Loa Observatory seemed to be the best facility to meet the needs of the project. Phone conversations were held with Mr. Elmer Robinson, the Director of MLO concerning the possibility of deploying and a site visit was made to the observatory while on travel to another deployment in June, 1991. Mr. Robinson indicated that, funding was limited, but that some arrangement might be possible by perhaps hiring a part time student to help take the measurements. Since we had no funding for support of the student we decided that the deployment would need to be delayed until another funding period. Mr. Robinson retired shortly after our site visit. We have had phone conversations with his successor, Dr. Russell Schnell who has a somewhat different impression of the Observatories ability to support non NOAA sponsored programs. Dr. Schnell explained that it would be necessary to hire a technician to support our deployment and he estimated the annual cost at $\$ 25 \mathrm{~K}$.

In the same time frame it had come to our attention that solar interferometric measurements are currently being made at MLO by Denver University, and that the new laboratory facility proposed for the observatory includes a slot for an emission interferometer by the same group, making additional data collection a MLO redundant. Therefore, the decision was made to deploy as opportunities allowed until the situation at MLO becomes better defined.

The first opportunity for a deployment was during WISP (WInter Storms Project) held in February and March, 1991 at Platteville, CO which allowed an intercomparison with the system from the University of Wisconsin. The only opportunity for a high altitude deployment to date was during the late summer of 1991 at Pingree Park, a mountain site at $3 \mathrm{~km}$ elevation which is one of the Colorado State University campuses. This deployment was in our original project planning as a checkout before any tropical deployment was attempted. There followed an opportunity during the second FIRE (First ISCCP (International Satellite Cloud Climatology Project) Regional Experiment) cirrus IFO (Intensive Field Observation) activity at Parsons, Kansas. The most recent opportunity was $F$ asented by the second marine stratocumulus IFO during ASTEX (Atlantic Stratocumulus Transition EXperiment) on the Island of Porto Santo near Madeira during June, 1992. The following paragraphs summarize the progress toward the project goals during each of these deployments.

\section{2a WISP Deployment}

The first opportunity to deploy the system in a more or less fully instrumented field program was during the WInter Storms Project (WISP) which took place in February and 
March of 1991. It was during WISP that the thermoelectrically cooled blackbodies were found to be susceptible to frost deposition a condition exasperated by high surface wind gusts which introduced moisture into the protected blackbody enclosures. The aperture of the closed system had to be temporarily uncovered to view the atmosphere. The deposition of frost introduced an uncertainty in the emissivity of the cooled black body. During WISP an opportunity was provided to compare results with those of the University of Wisconsin. These preliminary comparisons revealed discrepancies in the retrieved spectra, especially in the atmospheric window region. The disagreement was attributable in part to differing spectral resolutions and differing processing techniques; however, the discrepancies withstood closer scrutiny. The frost induced change in the emissivity of the cold blackbody was the most likely cause.

\section{2b Pingree Park Deployment}

In order to observe signals of climate change, measurements of the natural atmosphere are needed; i.e., the atmosphere free of daily changes introduced by human activity. Also, because it is the upper portion of the troposphere and the lower portion of the stratosphere which is of prime interest, a high elevation site was dictated. These were the reasons the deployment was originally planned for Mauna Loa Observatory. Pingree Park is one of the campuses of CSU and is located 50 miles southwest of the main campus at an approximate elevation of 9500 feet. This deployment was originally proposed prior to that at Mauna Loa as a checkout of the systems capabilities.

The interferometer was taken to the mountain site in August, 1991 in a field laboratory bus. A radio theodolite sounding system was also taken to the site. The system remained at the site until early October, 1991. Spectra were collected every few days under a variety of sky conditions; however, it wasn't until early fall that frequent clear sky conditions prevailed.

\section{Zenith Angle Dependence}

As a general rule spectra were collected as a function of observation zenith, usually starting at $0^{\circ}$ and proceeding at each $15^{\circ}$ of zenith to $75^{\circ}$. An atmospheric sounding was taken during the period of the zenith angle scans. Fig. 4 is an example of a sequence of spectra taken at different observation zenith angles under clear sky conditions.

Several interesting features pertaining to temperature retrieval are evident in the figure. First, the plot indicates those regions (generally the less opaque regions) which are most sensitive to changes in observation zenith. The $\mathrm{CO}_{2}$ emission region between 732 and $740 \mathrm{~cm}^{-1}$ shows a marked dependence on zenith angle. This is the region in which the original sensitivity to ratios of radiances was noted. Other portions of the spectrum, such as the near opaque region near $720 \mathrm{~cm}^{-1}$, indicate very little sensitivity to the change in observation zenith. In these regions the radiance has been emitted from the lower 


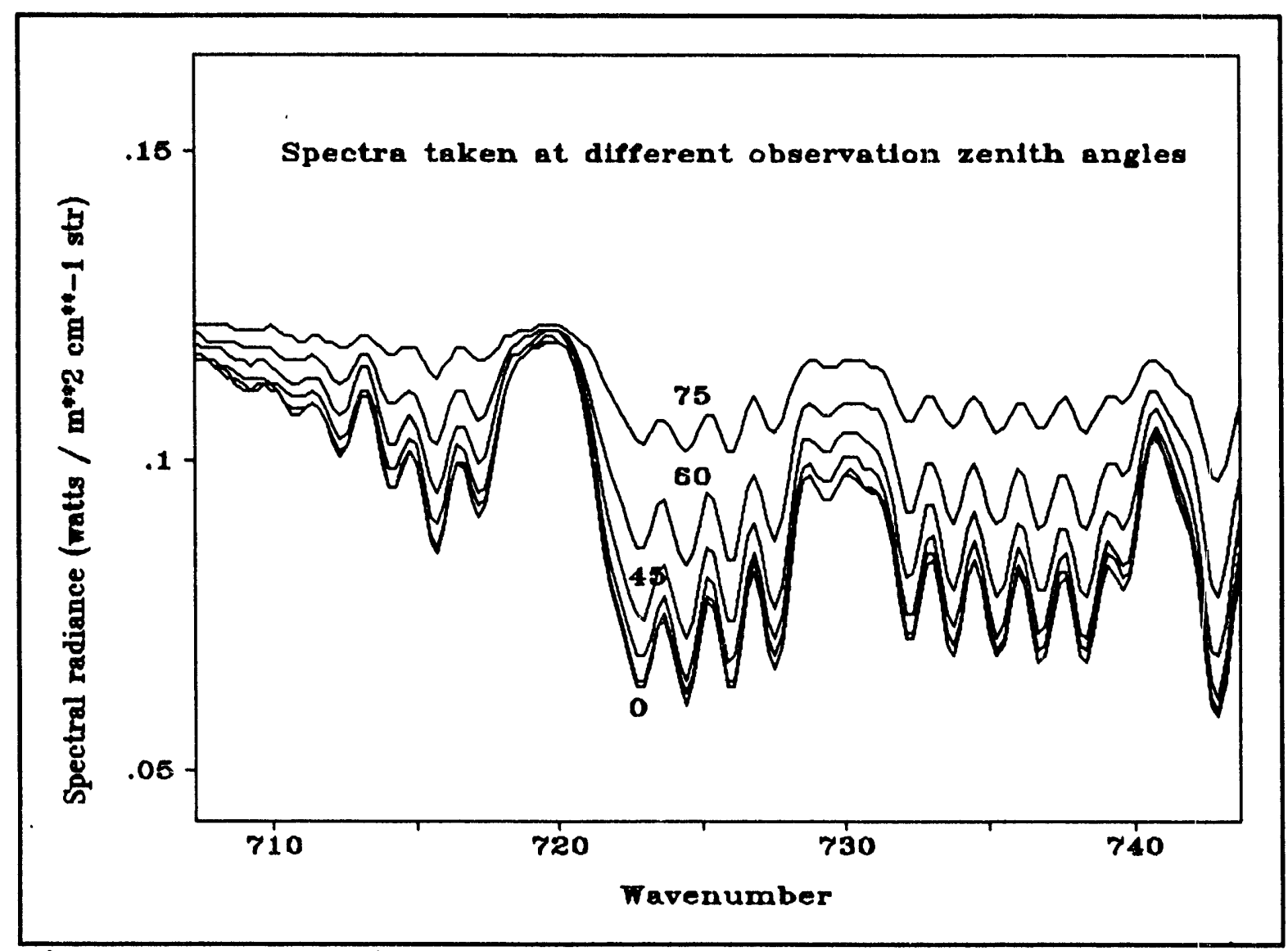

Figure 4. Spectra collected at observation zenith angles of $0,15,30,45,60$ and $75^{\circ}$ under clear sky conditions.

atmosphere and increases in the optical path result in a less dramatic increase in radiance. While the ratio of emitted radiances has been shown to contain information on the concentration of $\mathrm{CO}_{2}$, and to a lesser extent information about the mean temperature of the atmosphere, retrievals may or may not benefit from data collected at the greater zenith angles. The bulk of the information for retrieving temperature lies in the spectral resolution with which the data are collected. For example, currently at a resolution of $1.0 \mathrm{~cm}^{-1}$, over 1500 measured spectral radiances are available for the inversion algorithm. From Fig. 4 it is apparent that, especially in the $75^{\circ}$ case, some spectral smoothing is evident compared to the $0^{\circ}$ case. On the other hand, it may be that in other spectral regions, the emission from weak lines is enhanced at the higher elevation angles to a point above the noise level so that they add information content to the retrieval process. This aspect of the retrieval requires further investigation. At this point it appears that spectra collected at different zenith angles will be most useful in providing information on concentration of $\mathrm{CO}_{2}$ or as a method of detection of cirrus clouds as described next. 


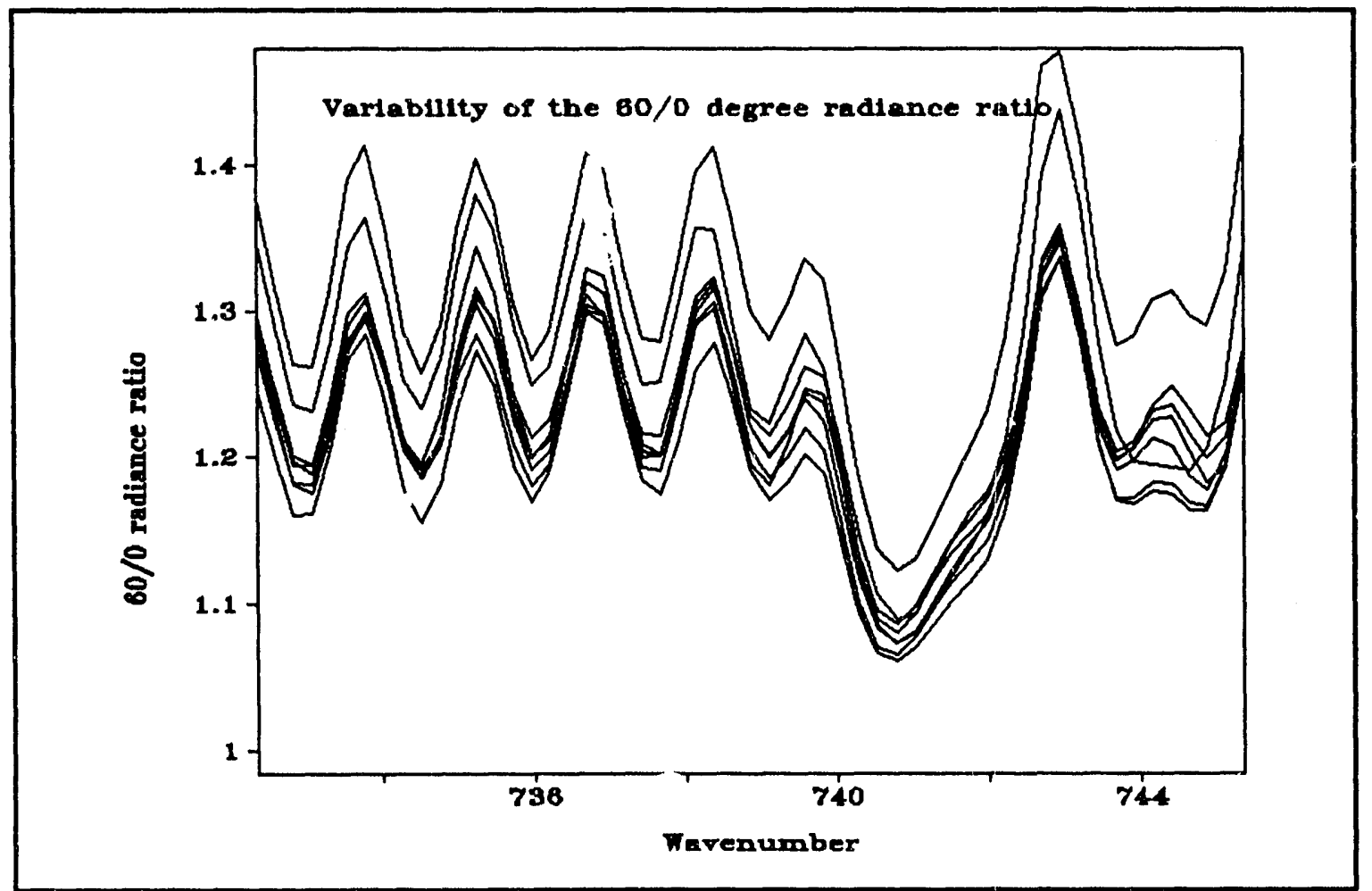

Figure 5 Ratios of $60^{\circ} / 0^{\circ}$ observed radiances under clear sky and thin cloud conditions.

\section{Cirrus Cloud Detection}

Since the $60^{\circ} / 0^{\circ}$ radiance ratios are sensitive to changes in the concentration of $\mathrm{CO}_{2}$, it is important to establish the precision at which the ratio can be measured for constant concentration of the gas. Eight cases were examined to establish the baseline precision for measurement of the ratios. Fig. 5 is a plot of the ratios for these cases in the region initially found to be most sensitive to the $\mathrm{CO}_{2}$ concentration.

First, it should be noted that the top two curves in the figure resulted from data collected under thin cirrus overcast conditions. In the top curve a thin cirrus deck was obvious and for the second curve from the top, nearly sub-visual cirrus conditions prevailed. Actually, the top tw.J curves resulted from sequential spectra collection under a dissipating cirrus layer, and it was only by observing the portion of the sky near the solar disk that any indication of cloud particles could be visually detected for the second curve. The spectra for the dissipating cirrus deck is discussed further below. The ratios for the truly clear sky cases are remarkably constant as shown in Table 1 in which the value of the ratios at a wavenumber of $745.846 \mathrm{~cm}^{-1}$ for all eight cases of Fig. 5 . 
The consistency of the ratio, even though the data set is small, is encouraging. The standard deviation of the clear sky value is less than $0.5 \%$ of its mean. This type of ratioing is, of course, sensitive in part to temperature structure. The range of surface temperature deviation amongst the clear sky cases represented in the Table was only 3.7 $\mathrm{C}$ and the deviations in upper air temperatures were even smaller. Thus, further evaluation of the dependence on temperature structure is indicated. Also this preliminary data indicates that the ratio may be sensitive to the presence of even extremely thin clouds. Clearly, more observations are necessary to establish the usefulness of these ratios.

\begin{tabular}{|c|c|c|}
\hline Date & Sky condition & Ratio Value \\
\hline $9 / 03 / 91$ & clear & 1.431 \\
\hline $9 / 05 / 91$ & near sub-vis cirrus & 1.533 \\
\hline $9 / 16 / 91$ & cirrus & 1.624 \\
\hline $9 / 16 / 91$ & clear & 1.431 \\
\hline $9 / 16 / 91$ & clear & 1.435 \\
\hline $9 / 25 / 91$ & clear & 1.425 \\
\hline $9 / 25 / 91$ & clear & 1.443 \\
\hline $9 / 25 / 91$ & clear & 1.435 \\
\hline
\end{tabular}

Table 1. Values of the $60^{\circ} / 0^{\circ}$ radiances at $745.846 \mathrm{~cm}^{7}$ for clear or nearly clear conditions.

One of the primary signals of climate change would be a change in the amount or type of cloud cover. This topic is at the core of the warming scenario. Although the ratio method above seems to be sensitive to the presence of even the thinnest clouds, it is not viewed as the method with the most potential to diagnose cloud properties. Rather the radiometric signal in the atmospheric window region is seen as containing the most information about the cloud type.

An interesting opportunity to observe the effect of upper level clouds on the window spectra presented itself on September 5, a day which began with moderately thick cirrocumulus overcast. By late morning the clouds had thinned considerably to what could be termed thin cirrus. Early in the afternoon the clouds could almost be termed sub-visual cirrus, and it was only by looking near the solar disk that some cloud was evident. Fig. 6 depicts the spectra at $0^{\circ}$ observation angle for each of these cases. The downward slope of the spectra from low to higher wavenumbers is evident in the figure. The two bottom curves are portions of the spectra from which the ratios for the two cloudy cases of Table 1 were extracted. 


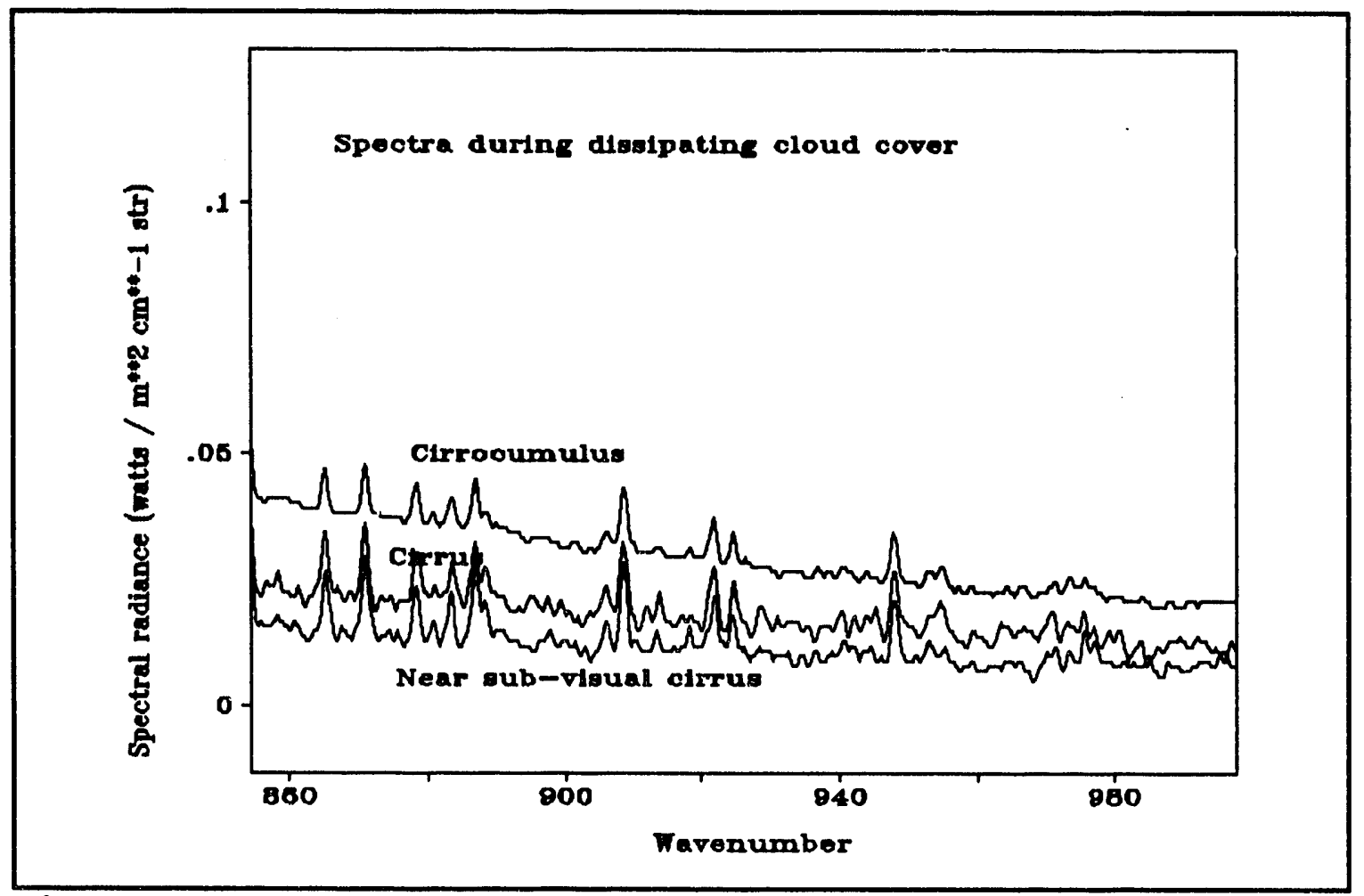

Figure 6. Downwelling spectral radiance in the atmospheric window during conditions of dissipating cirrus overcast.

Of course, in addition to the mere presence of clouds, their physical characteristics are subject to change as well in any climate change scenario. The primary cloud parameter of interest is the cloud optical depth. Also important is the microphysical makeup of the cloud. Another example of the slope in the atmospheric window attributable to cirrus may be found in the next section describing the deployment at the second cirrus FIRE IFO as well as a derivation of particle equivalent radius.

\section{2c Fire IFO Deployment}

The next opportunity for system deployment was during the second FIRE cirrus IFO. The department of Atmospheric Science at Colorado State University brought an ensemble of instrumentation to Parsons KS, about 60 miles from the experiment hub at Coffeyville. The instrument was housed in an enclosure to reduce any short term temperature variations of the interferometer. Throughout the last half of November and early December, 1991 over a hundred spectra were collected under clear and overcast conditions. This deployment provided an excellent opportunity to test the sensitivity of the window slope for the purpose of detection of cirrus cloudiness since 
a lidar supplied by NASA Langley was located within $10 \mathrm{~m}$ of the interferometer. Fig. 7 is a plot of uncalibrated lidar backscatter taken on November 26, 1992. Evident in the figure is the approaching cirrus layer.

Fig. 8 is a plot of two spectra, one taken at $1615 \mathrm{Z}$ before the cirrus and the upper spectrum taken at $1730 \mathrm{Z}$ after the leading edge of the cirrus had arrived. Again the system's sensitivity to the cloud layer is demonstrated.

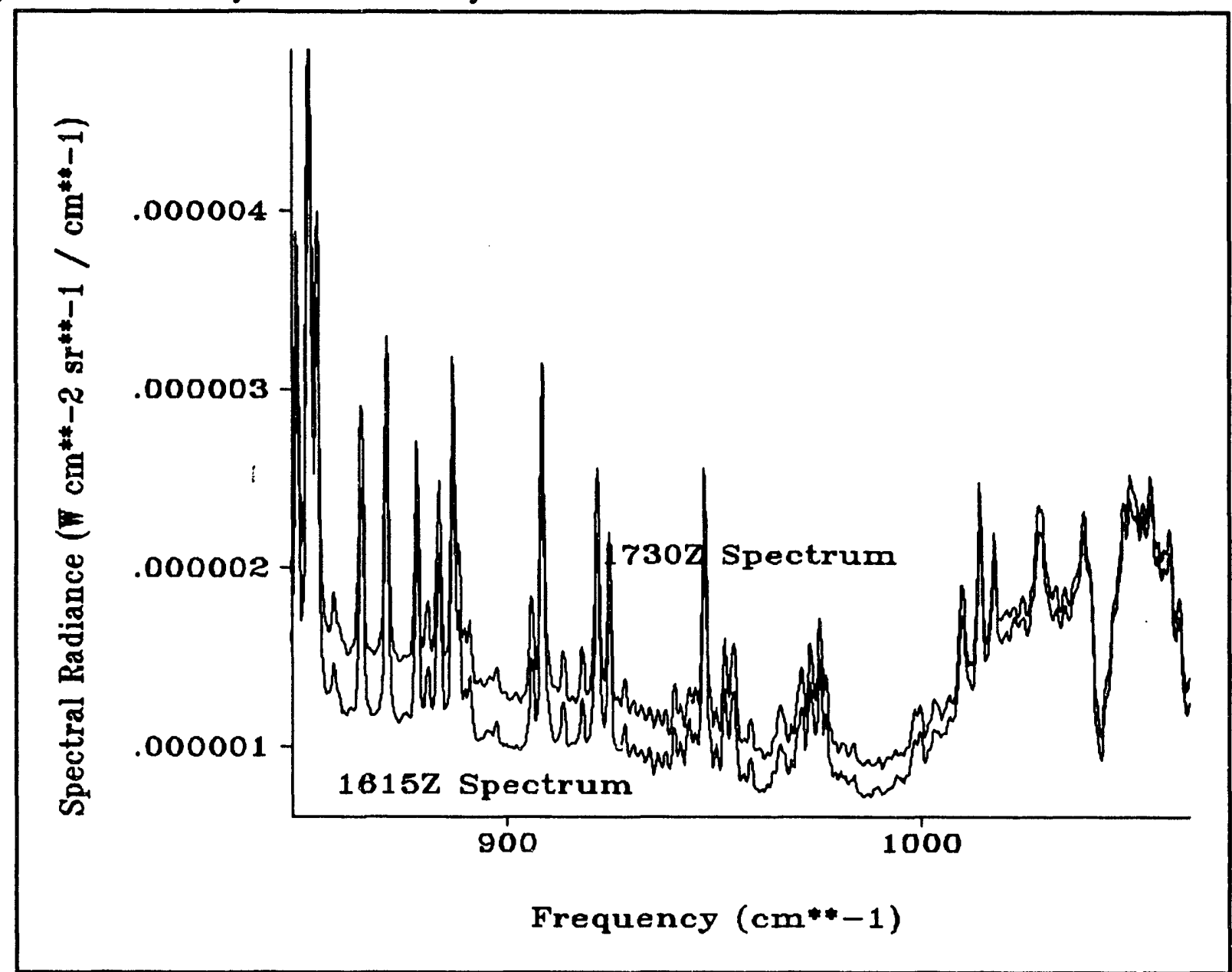

Figure 8. Spectra in a portion of the atmospheric window after cirrus onset (upper) and before cirrus onset (lower).

\section{Estimation of Effective Particle Size}

The influence of cirrus cloudiness on the emission in the window region is clearly evident from the plots shown above. Recently, the ability to estimate the radius of equivalent spherical particles has been demonstrated by examining the slope of the emission in the window region; see Prabhakara (1988), Ackerman et al (1990), and 


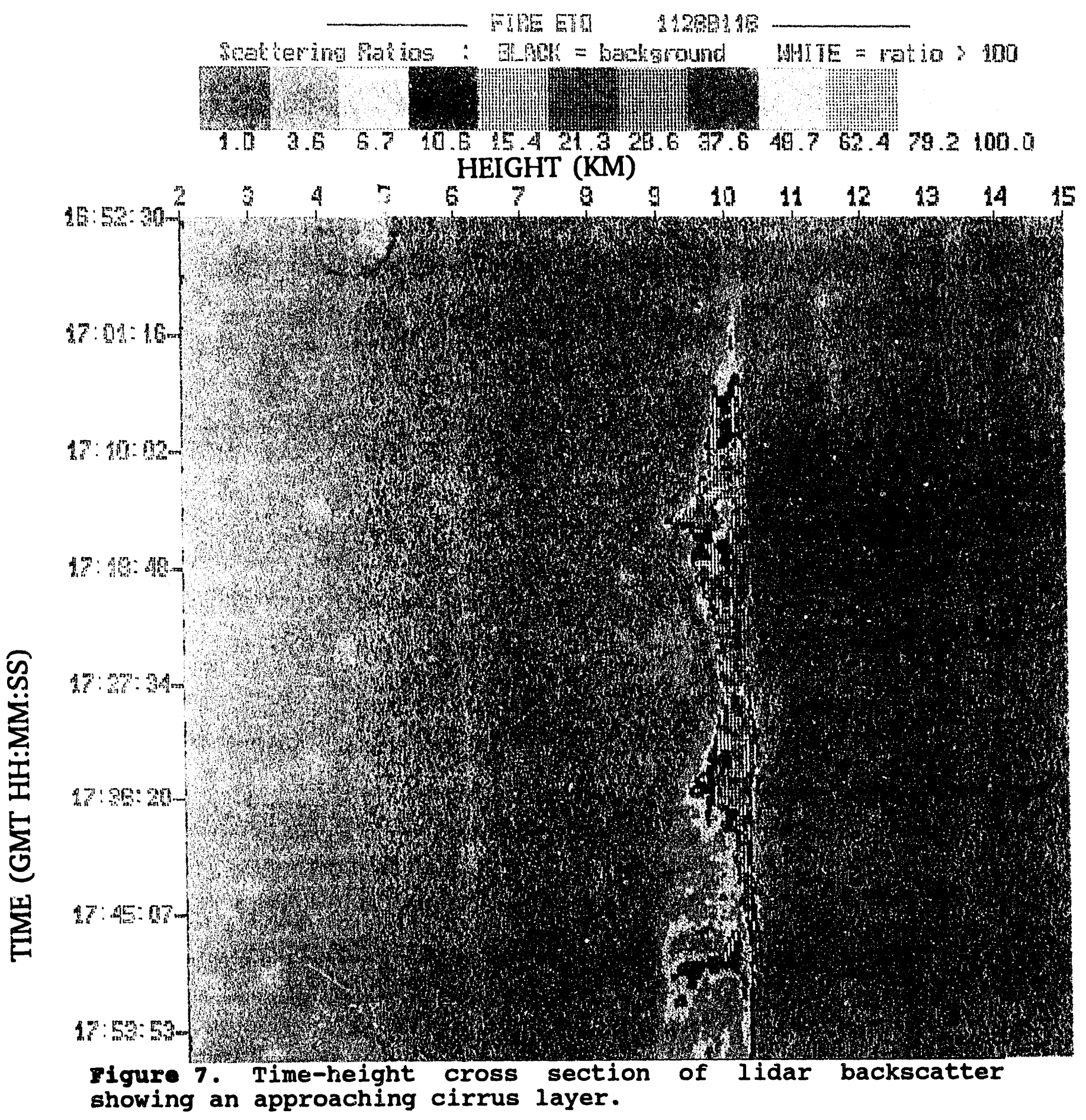


Parol et al (1991). The theoretical basis for this determination relies on the sensitivity of the ratio of the absorption efficiency factor at two wavelengths to particle size. For example, writing a simplified equation for the radiance below the cloud $\mathrm{I}_{\mathrm{ob}}$ as;

$$
I_{\mathrm{obs}}-I_{c 1 \mathrm{x}}+B\left(T_{c}\right)\left[1-e^{-\tau^{*} / \mu}\right] T_{\mathrm{atm}}
$$

where $I_{\text {ctr }}$ is the downward radiance emitted by the atmosphere below the cloud which is approximated by measuring the downward radiance in a clear atmosphere, $T^{*}$ is the optical thickness of the cloud, $\mu$ is the cosine of the viewing angle, and $B\left(T_{c}\right)$ is the Planck function at the cloud temperature $T_{c}$, and $T_{\text {dum }}$ is the transmission of the atmosphere beneath the cloud, where it has been assumed that the cloud is isothermal and scattering has been ignored. Defining $\Delta I-I_{a b s}-I_{c l r}$, and solving for $\tau^{*}$ gives;

$$
\tau^{*}=-\ln \left[1-\frac{\Delta I}{T_{a t m} B\left(T_{c}\right)}\right] .
$$

The optical thickness may be evaluated at two wavenumbers in this manner and the ratio $\gamma-\tau^{*}{ }_{1} / \tau^{*}{ }_{2}$ formed. For a monodispersed cloud composed of spherical particles of radius a, the optical thickness may be written as, $\tau^{*}-\pi N_{0} a^{2} Q_{a b s}$, where $N_{0}$ is the concentration of the particles and $Q_{a b}$ is the absorption efficiency facior. The ratio of optical depths is then given by, $\gamma-\frac{Q_{a b B, 1}}{Q_{a b s, 2}}$. Using single scattering algorithms, either for Mie scattering for spherical particles or more sophisticated methods such as the Discrete Dipole Approximation for crystals of various shapes, it is then possible to plot the ratio as a function of particle size as was done in Parol et al (1991). This exercise was carried out for the same cirrus cloud episode, the leading edge of which is shown in the lidar plot above. The results indicated that at a time of thin cirrus at $1800 \mathrm{Z}$, the equivalent spherical particle radius was $10.4 \mu \mathrm{m}$ and after the cloud thickened a value of $23.8 \mu \mathrm{m}$ was derived.

\section{2d ASTEX Deployment}

The most recent deployment of the system took place during June, 1992 on the Island of Porto Santo, Madeira during the ASTEX activity. The deployment presented several opportunities for collecting spectra of clear sky as a function of zenith angle, of subtropical cirrus and of the marine stratocumulus overcast. These data were collected in conjunction with three hourly rawinsonde launches and hourly RASS soundings of the lower troposphere. The primary value of these data will be to provide a detailed 
thermodynamic record of the atmosphere which will be used to verify the temperature retrieval analysis described below.

\subsection{Temperature Retrieval Algorithm}

As indicated in the original proposal, the ratio of $60^{\circ}$ to $0^{\circ}$ radiances shows sensitivity mainly to the concentration of the greenhouse gases and a smaller sensitivity to temperature structure. The alternative method of detecting changes in the temperature structure is the more conventional temperature retrieval or inversion approach. The basic question about the effectiveness of conventional temperature retrieval in this application is the height to which the temperature structure can be retrieved from ground based interferometric measurements. Initial retrieval from interferometric measurements by Smith et. al. (1988), indicates that temperature retrieval may be achieved to an altitude of $500 \mathrm{mb}$ and water vapor to $700 \mathrm{mb}$, based on comparisons with satellite data. These results were reported for data collected at Madison, WI. It is our intention to apply a similar algorithm to the data collected at the Pingree Park site to see if the high altitude collection site extends the height to regions where the $2 \mathrm{CO}_{2}$ atmosphere temperature structure change is predicted to be most prominent.

Selection of an appropriate retrieval algorithm was, of course, the first step into this phase of the research. There are various approaches to the problem, and the selection of a particular algorithm was based on the following attributes of such algorithms in general. First, the algorithm should be based on physical modeling rather than statistical correlation. In the latter category we also cast the "neural network" or "artificial intelligence" approach. It is important to use a physically correct model since in a climate change scenario, past statistical data bases would not necessarily be representative of current conditions. Also, the need for a large statistical database is avoided. Second, the approach should solve the true nonlinear system of equations. Linearization of the equations is less accurate and thus, less preferable. Third, the algorithm's solution should not be sensitive to the order in which parameters are considered. For example, if the algorithm's solution is dependent upon the order in which clouds and $\mathrm{H}_{2} \mathrm{O}$ vapor are treated, then past experiences with the model, which are almost certainly tuned to the downward looking problem, will not necessarily provide optimal results for this application. Fourth, the model should be generally compatible with FASCOD3. We have based all of our modeling effort on the FASCOD3 code. Thus, a retrieval algorithm not compatible with the features of FASCOD3 would require an additional investment of resources. Finally, the question of availability was considered. Creation of a new retrieval algorithm was not one of the research goals of this effort. Thus, a code which was available received high consideration. In consideration of these requirements we have acquired from Phillips Laboratory (formerly AFGL) the Path Characterization Algorithm for FASCODE. 
The implementation of the Path Characterization Algorithm with FASCOD3 has not been at all straightforward. In fact, due to the manner in which FASCOD3 implements calculation of the Planck function, the retrieval algorithm may not be totally compatible with FASCOD3. This is due to a feature in FASCOD3 which weights the calculation of emitted radiance based on temperatures at the boundaries of the atmospheric layers while the retrieval algorithm uses a mean layer temperature to calculate derivatives for the forward model and perform the inversion. We have invested a considerable amount of effort attempting to implement the retrieval algorithm with a pre-beta version of FA.SCOD3. It appears that the retrieval algorithm is functioning with our version of FASCOD3 although these results are very preliminary. For example, Fig. 9 shows the results of a "simulated" case in which an upward looking detector views an atmosphere characterized by a lower level inversion. The plot shows the differences ("measurements"-reference) and (retrieval "measurements"). The term "simulated" retrieval implies that the data are generated artificially using FASCOD3. In this case no instrument noise has been introduced. In the plot the term "measurements" refers to simulated data. In order to simulate the measurements in this case the temperature in the second and third layers of the atmosphere have been increased by 6 and $2 \mathrm{~K}$ with respect to the reference profile. The dashed line indicates the accuracy of the retrieval.

In order to obtain a retrieval from actual data it is necessary to match the characteristics of the forward modeled data to the measurements. Routines have been incorporated into the Path Characterization Algorithm to introduce the characteristics of the Bomem MB100 interferometer. Basically, the Path Characterization Algorithm runs FASCOD3 as a subroutine in order to calculate a spectrum from a guess or reference atmospheric profile. Subsequently, derivatives of the radiance field are calculated to determine the sensitivity of the radiance field to changes in the parameter being retrieved. In our version of the algorithm, FASCOD3 is executed as a subroutine using a rectangular scanning function output at a resolution of $0.05 \mathrm{~cm}^{-1}$ in order to simulate a radiance field at high resolution. The data are operated on using an FFT in order to create a modeled interferogram. The model interferogram is truncated to decrease the resolution to that of the instrument and apodization effects are introduced. Trailing zeros are added before another FFT operation in order to FFT smooth the modeled data in the same manner as the calibration program processes the measured data. Graphical comparison indicates a good match between modeled and measured data, but as of the writing of this report, we have not successfully retrieved an actual profile. It is not clear at this time if there is a still a basic problem with the Path Characterization Algorithm or if our instrument "matching" software requires further refinement. 


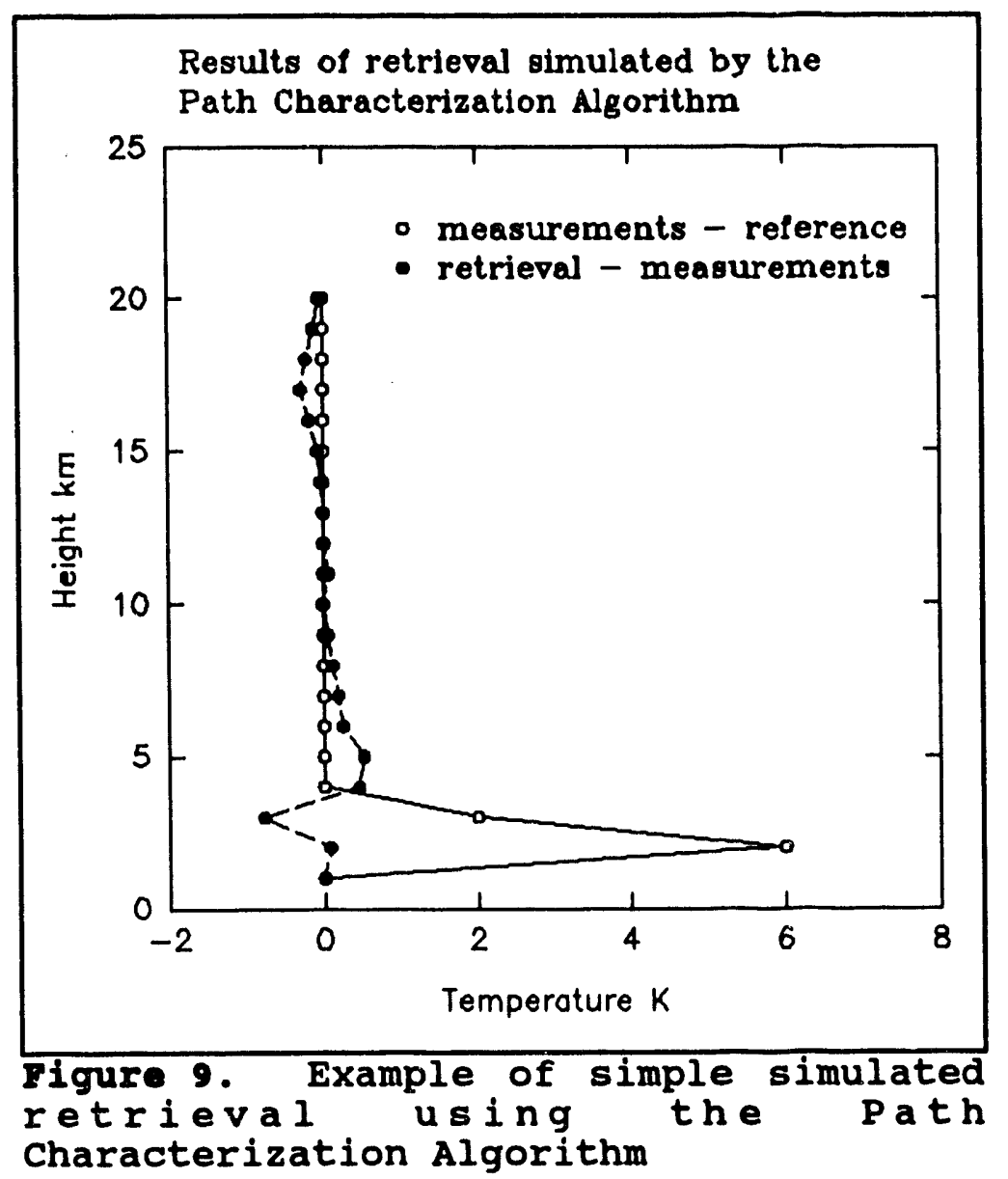

\subsection{Summary}

A system designed to collect data signaling possible climate change has been assembled. Two calibration configurations have been tested and the LN2-ambient temperature black body system has been selected for continued operation. The system was deployed during WISP and spectra compared to simultaneous spectra taken by the University of Wisconsin. Discrepancies suggested the alternate calibration method. During the summer of 1992, the system was deployed at a high elevation site in the mountains west of the main campus of Colorado State University. Spectra and atmospheric soundings were collected under various sky conditions. The results show favorable comparisons with the FASCOD3 model. Spectra were collected at a different observation zenith angles to investigate those portions of the spectrum most sensitive to changes in atmospheric path. The stability of the $60^{\circ} / 0^{\circ}$ radiance ratios have been evaluated for the small number of clear sky spectra collected. Initial results indicate that the ratio is constant to about $0.5 \%$, (excluding large variations in 
the temperature structure) for clear sky cases. The ratio also shows sensitivity even to thin cloudiness. Examination of the spectra in the atmospheric window region shows that the slope of the floor of the spectra is sensitive even to near sub-visual cirrus conditions. The system was deployed at the 2nd FIRE cirrus IFO at Parsons, KS. Over 100 spectra were collected under clear sky and cirrus overcast conditions. The sensitivity to the slope of the window region spectra were again seen for cirrus confirmed by nearby LIDAR. A simple method to derive equivalent spherical radius has been demonstrated. The system was deployed during the June, 1992 ASTEX activity. Spectra of sub-tropical cirrus, marine stratocumulus and clear sky as a function of zenith angle were collected. A temperature and gaseous concentration retrieval algorithm has been acquired and incorporated with the line by line transmission algorithm FASCOD3 in order to investigate application of the more conventional inversion to the current problem. At the present time it appears the algorithm is functioning correctly in a theoretical retrieval mode. A high speed computer workstation has been acquired to facilitate this phase of the research.

\subsection{References}

Ackerman, S. A., W. L. Smith, J. Spinhirne, H. E. Revercomb: Spectral properties of cirrus clouds in 8-12 micron window, FIRE Science Team Workshop, NASA, in press 1989.

Griffiths, P. R., and J. A. de Haseth, 1986: Fourier Transform Infrared Spectrometry, John Wiley and Sons, New York, Vol 83, 656 pp.

Parol, F., J. C. Buriez, G. Brogniewz, and Y. Fouquart, 1991: Information content of AVHRR channels 4 and 5 with respect to the effective radius of ciffus cloud particles, J. Appl. Meteor.30, 923-984.

Prabhakara, C., R. S. Fraser, G. Dalu, Man-Li C. Wu, and R. J. Curran, 1988: Thin cirrus clouds: Seasonal distribution over oceans deduced from Nimbus-4 IRIS. J. Appl. Meteor., 27, 379-399.

Revercomb, H. E., H. Buijs, H. B. Howell, D. D. Laporte, W. L. Smith, and L. A. Sromosvsky, 1988: Radiometric calibration of IR Fourier transform spectrometers: solution to a problem with the High-Rsolution Interferometer Sounder. J. Appl. Opt.,27,3210-3218.

Rind, D. 1987: The doubled $\mathrm{CO}_{2}$ climate: Impact of the sea surface temperature gradient. J. Atmos. Sci. 21, 3235-3268.

Smith, W. L., M. J. Lynch, H. E. Revercomb, R. Knuteson, C. M. Hayden, H. M. Woolf, D. LaPorte, and H. Buijs: A low-cost ground-based temperature, humidity, and 
trace gas monitoring system. Proceedings of conference entitled, "Remote Sensing of the Lower Troposphere", May 31- June 3, 1988, Boulder, CO. Jointly sponsored by AMS/NOAA/NCAR. 

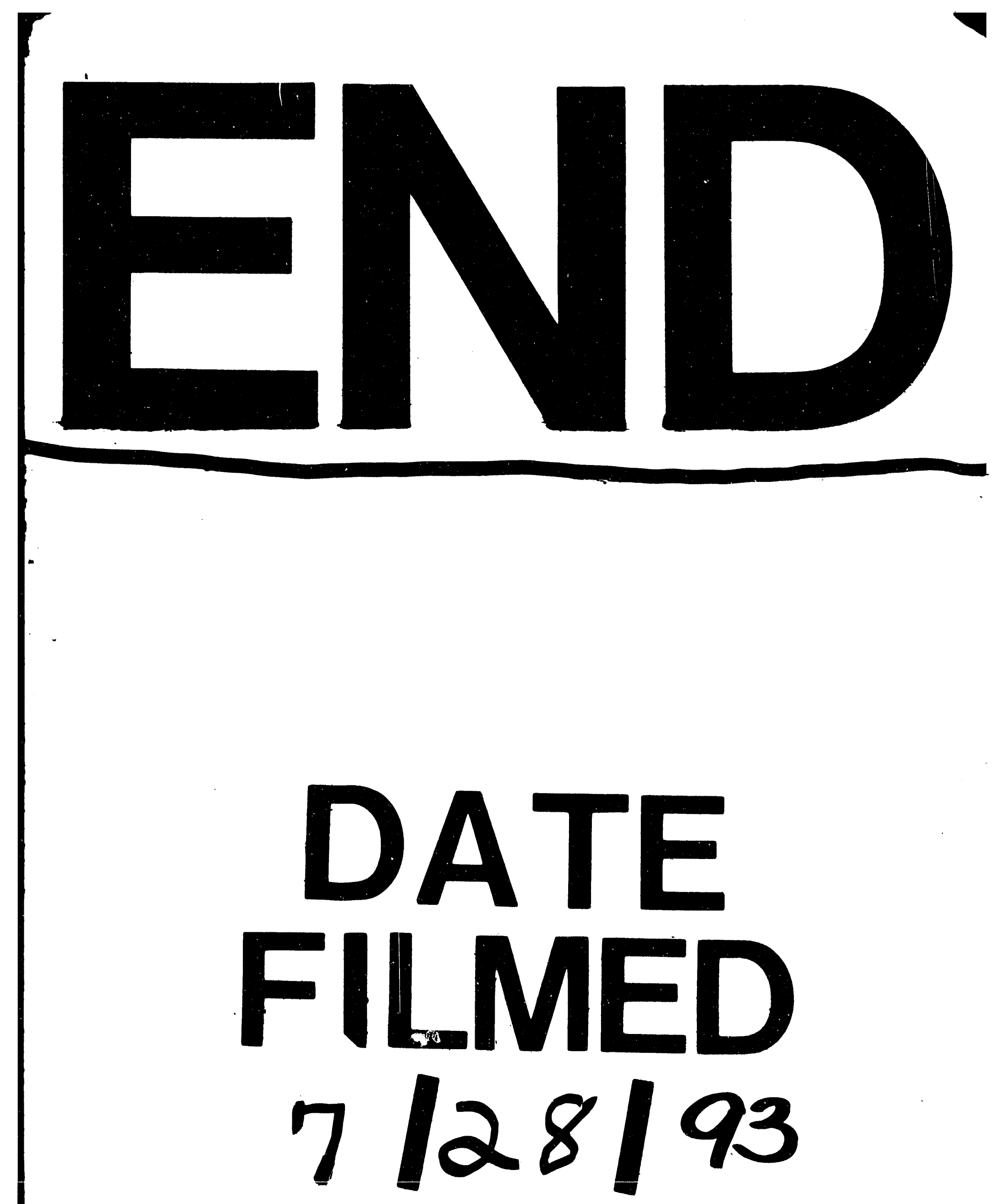
